\title{
A nearly closed ballistic billiard with random boundary transmission
}

\author{
Igor Rozhkov, Ganpathy Murthy \\ Department of Physics and Astronomy, University of Kentucky, Lexington, Kentucky 40506
}

\begin{abstract}
A variety of mesoscopic systems can be represented as billiard with a random coupling to the exterior at the boundary. Examples include quantum dots with multiple leads, quantum corrals with different kinds of atoms forming the boundary, and optical cavities with random surface refractive index. We study an electronic billiard with no internal impurities weakly coupled to the exterior by a large number of leads. We construct a supersymmetric nonlinear $\sigma$-model by averaging over the random coupling strengths between bound states and channels. The resulting theory can be used to evaluate the statistical properties of any physically measurable quantity. As an illustration, we present results for the local density of states.
\end{abstract}

When the size $L$ of a two-dimensional (2D) mesoscopic structure is reduced beyond the elastic mean free path $l$ of the electrons, all the scattering takes place at the boundary. An electron may escape through the boundary, get reflected back specularly, or participate in both processes. Besides quantum dots [1], which can exhibit both diffusive and ballistic behavior [2, 3], there are mesoscopic billiards which are ballistic by construction. Examples include quantum corrals (QC) [4], optical corrals [5], optical resonant cavities [6], and the artificial atoms proposed in Ref. 7].

A quantum dot has boundary losses, unless the confining potential is chosen to be infinitely high. Such losses are usually modeled by coupling to a number (possibly infinite) of open channels [8], although the precise details of coupling are generally unknown. In this paper we focus on internal ballistic dynamics of a clean circular dot which is nearly closed, i.e., it is weakly coupled to a large number of leads. The crucial ingredient in our model is the randomness of the transmission coefficients. It enables us to carry out an ensemble average using the supersymmetry method [9], and, as a side-benefit, acts as a natural regularizer, helping us avoid the technical difficulties of previous supersymmetric approaches to closed ballistic systems 3,10$]$. The resulting theory is a "surface" $\sigma$-model, which resembles the conventional diffusive $\sigma$-model [9], but has the "diffusion" modes confined at the boundary of the dot. These can be associated with classical whispering gallery trajectories, which run along the walls of the system and are known to strongly influence transport through mesoscopic structures [11].

Furthermore, as pointed out in Ref. 12], the ballistic analogs of density relaxation modes in diffusive samples originate from trajectories which remain close to each other in configuration space. Given the almost closed nature of our dot, the whispering gallery modes (WGM) 11] are expected to impact the long-time characteristics of the internal dynamics and to play a major role in quantum interference effects. By contrast, the "star-like" and "asterisk" trajectories, which approach the lead mouth at small angles to the boundary normal and are more likely to exit the billiard, cannot contribute 13 to the response functions. In other examples [4, 7] the electrons do not decay into well-defined leads, but because of the significant similarities between the three nanostructures our approach will apply, mutatis mutandis, to them as well. To be specific, the long-lived modes which play a chief role in their internal dynamics have the same classical origin.

A quantum dot in which electrons are confined by a hard wall potential but can escape into leads 14] can be described by the non-Hermitian effective Hamiltonian

$$
H_{\text {eff }}=\mathcal{H}_{0} \mp \frac{i}{2} \sum_{n=1}^{N_{\text {leads }}} \widehat{B}_{n} \delta_{C_{n}}
$$

Here, $\mathcal{H}_{0}$ is the Hamiltonian of the closed quantum dot, and $\delta_{C_{n}}$ is a surface $\delta$-function with uniform support on the crossection $C_{n}$ which separates the $n$th lead from the billiard. It is important to note that the leads have been "integrated out". In other words, one replaces an open dot with plane wave boundary conditions in the asymptotic region and Dirichlet conditions along the rest of the boundary, with a closed dot and simplified boundary conditions [14]. The operators $\widehat{B}_{n}$ are defined through their action on an arbitrary function

$$
\widehat{B}_{n} \Psi(\mathbf{r})=\int_{C_{n}} \sum_{i}^{M_{n}^{\text {channels }}} \gamma_{n}^{(i)} v_{n}^{(i)} \varphi_{n}^{(i)}(\mathbf{r}) \varphi_{n}^{(i)}\left(\mathbf{r}^{\prime}\right) \Psi\left(\mathbf{r}^{\prime}\right) d \mathbf{r}^{\prime},
$$

where $\gamma_{n}^{(i)}<1$ is a coupling coefficient, $v_{n}^{(i)}$ is a transverse velocity, and $\varphi_{n}^{(i)}$ is a normalized eigenfunction of transverse motion for $i$ th channel in $n$th lead.

Thus, the solution of the original open dot problem reduces to the solution of

$$
\left(E-H_{e f f}\right) G^{R, A}\left(\mathbf{r}, \mathbf{r}^{\prime}\right)=\delta\left(\mathbf{r}-\mathbf{r}^{\prime}\right),
$$

for the retarded (advanced) Green's function $G^{R, A}$, with Neumann boundary conditions $\left.\left(\mathbf{x}_{n} \mathbf{v}_{r}\right) G^{R, A}\left(\mathbf{r}, \mathbf{r}^{\prime}\right)\right|_{C_{n}}=$ 0 , where $\mathbf{x}_{n}$ is a unit vector parallel to the waveguide walls, and $\mathbf{v}_{r}$ is the velocity operator. It acts from the side of the lead.

Just as in studies of chaotic scattering [15], the key tool in calculations of response functions of ballistic (or disordered) systems with leads is the operator $\left(E-H_{e f f}\right)^{-1}$. 
In the framework of the supersymmetry method it can be treated in the same way as $\left(E-H_{0}\right)^{-1}$, as was done in Ref. 14], where nonperturbative calculations were carried out for disordered dots. Note, that we pursue the non-universal, i.e. non-"random matrix theory" regime.

For a ballistic dot the dynamics is governed by the operator: $\mathcal{H}_{0}=(\mathbf{p}-(e / c) \mathbf{A})^{2}$, where we assumed no potential and a constant magnetic field. The latter is introduced to break time reversal symmetry, which simplifies the application of the supersymmetry method, and makes the illustration of our approach more transparent.

We intend to calculate averages over the Gaussian distribution of dimensionless coupling coefficients $\gamma_{n}^{(i)}$. The relation of these coefficients to sticking probabilities, transmission coefficients and other commonly used parameters can be found in Ref. [15] in the context of the Hamiltonian approach to chaotic scattering. More insight on the physical meaning of these coefficients can be gained from Refs. 16].

Before commencing our supersymmetric derivation, we make a few simplifying assumptions. These assumptions can all be relaxed without affecting the physics, but are needed to simplify the technical details. We allow only one open channel in each lead and express the coupling coefficients as a sum of constant and stochastic parts: $\gamma_{n}=\bar{\gamma}+\widetilde{\gamma}_{n}$. For the statistics of $\widetilde{\gamma}_{n}$ we assume that $\left\langle\widetilde{\gamma}_{n}\right\rangle=0,\left\langle\widetilde{\gamma}_{n} \widetilde{\gamma}_{m}\right\rangle=x^{2} \delta_{n m}$, and that all higher moments factorize into second moments. Next, we "eliminate the leads" [14], passing to the Hamiltonian given by Eq. (11).

Upon introduction of four-component supervectors $\Psi(\mathbf{r})^{T}=\left\{S_{1}(\mathbf{r}), \chi_{1}(\mathbf{r}), S_{2}(\mathbf{r}), \chi_{2}(\mathbf{r})\right\}$, and supermatrices $L=\operatorname{diag}\{1,1-1,1\}, \Lambda=\operatorname{diag}\{1,1-1,-1\}$ (see, for example, Ref. 17]), the supersymmetric generating functional reads [18]

$$
\langle Z[J]\rangle_{\widetilde{\gamma}_{n}}=\int d \Psi^{*} d \Psi \mathrm{e}^{-\mathcal{L}[\Psi]}\left\langle\mathrm{e}^{-\mathcal{L}_{\delta}[\Psi]}\right\rangle_{\widetilde{\gamma}_{n}},
$$

where $\langle\ldots\rangle_{\widetilde{\gamma}_{n}}$ indicates averaging over random couplings to the leads

$$
\begin{aligned}
\mathcal{L}[\Psi] & =i \int \Psi^{\dagger}(\mathbf{r}) \widehat{\mathcal{H}} L \Psi(\mathbf{r}) d \mathbf{r} \\
& +\frac{\bar{\gamma}}{2} \sum_{n=1}^{N} v_{n} \int_{C_{n}} \Psi^{\dagger}\left(y_{n}\right) \varphi_{n}\left(y_{n}\right) \varphi_{n}\left(y_{n}^{\prime}\right) \Lambda L \Psi\left(y_{n}^{\prime}\right), \\
\mathcal{L}_{\delta}[\Psi] & =\sum_{n=1}^{N} \frac{\widetilde{\gamma}_{n} v_{n}}{2} \int_{C_{n}} \Psi^{\dagger}\left(y_{n}\right) \varphi_{n}\left(y_{n}\right) \varphi_{n}\left(y_{n}^{\prime}\right) \Lambda L \Psi\left(y_{n}^{\prime}\right) .
\end{aligned}
$$

with $\widehat{\mathcal{H}}=\widehat{\mathcal{H}}_{0} I_{4}+i \epsilon \Lambda,\left(\epsilon\right.$ is infinitesimally small) $\widehat{\mathcal{H}}_{0}=$ $-\nabla^{2} / 2 m-E, \varphi_{n}(y)=\sqrt{2 / d_{n}} \sin \left(\pi y / d_{n}\right)$ (for hard-wall lead of width $d_{n}$ ). Here $\int_{C_{n}}$ stands for a double integration over $y_{n}$ and $y_{n}^{\prime}$, the transverse coordinates along the crossection $C_{n}$ (perpendicular to the walls of a waveguide); the product $d y_{n} d y_{n}^{\prime}$ will be omitted in what follows.
Averaging over $\widetilde{\gamma}_{n}$ produces:

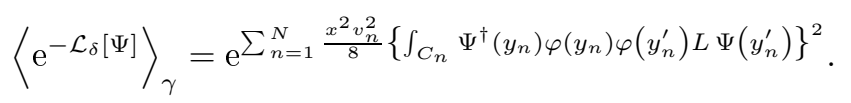

Then, using the Hubbard-Stratonovich transformation, we decouple the "interaction terms", introducing supersymmetric fields $Q_{n}\left(y_{n}, y_{n}^{\prime}\right)$ defined at each crossection $C_{n}$. To simplify the form of the action we further assume that the leads have identical width $d$ and are attached everywhere along the perimeter of the dot. We choose the total number $N=2 \pi R / d$ ( $R$ is the radius of the circle enclosing the dot) of leads to be large, and therefore $d \ll R$. We also assume identical transverse velocities: $v_{n}=v$. In the limit $N \rightarrow \infty$, the density field $Q$ becomes continuous. It turns into a function of a single variable the polar angle $\theta$.

After the Gaussian integration over $\Psi(\mathbf{r})$-variables, an important supermatrix which needs to be determined is the effective Green's function $\mathcal{G}\left(\mathbf{r}, \mathbf{r}^{\prime}\right)$. It satisfies

$$
\left(-\widehat{\mathcal{H}}_{0}-i \frac{m x v a}{2} \frac{\delta(r-R)}{r} \widetilde{Q}(\theta)\right) \mathcal{G}\left(\mathbf{r}, \mathbf{r}^{\prime}\right)=i \delta\left(\mathbf{r}-\mathbf{r}^{\prime}\right) \text {. }
$$

where $\widetilde{Q}(\theta)=Q(\theta)-(\bar{\gamma} / 2 x m d) \Lambda$ and $a=4 R d$. The generating function takes the form

$$
\langle Z[J]\rangle_{\gamma}=\int D Q \mathrm{e}^{F[Q]+F_{\epsilon}[\mathcal{G}]}
$$

with the free energy

$$
\begin{aligned}
F[Q] & =\operatorname{Str} \int d \mathbf{r} d \mathbf{r}^{\prime}\left\{-\frac{m^{2} a}{2} Q(\theta)^{2}\right. \\
& \left.\times \frac{\delta(r-R)}{r} \delta\left(\mathbf{r}-\mathbf{r}^{\prime}\right)+\ln -i \mathcal{G}^{-1}\left(\mathbf{r}, \mathbf{r}^{\prime}\right)\right\}
\end{aligned}
$$

and the symmetry breaking term $F_{\epsilon}[\mathcal{G}]=$ $-\operatorname{Str} \int \ln \left(I_{4}+\epsilon \Lambda \mathcal{G}\left(\mathbf{r}, \mathbf{r}^{\prime}\right)\right) d \mathbf{r} d \mathbf{r}^{\prime}$. The functional integration over $Q(\theta)$ is performed in saddle point approximation, which requires the solution of

$$
Q_{s p}(\theta)=\frac{x v}{2 m} \mathcal{G}\left(R, R, \theta, \theta, Q_{s p}(\theta)\right) .
$$

Assuming the solution to be diagonal and coordinate independent, we arrive at usual structure of the saddle point: $Q_{s p}(\theta)=Q_{0} \Lambda$. In order to analyze the fluctuations, the constant $Q_{0}$ and the diagonal Green's function supermatrix $\mathcal{G}_{s p}\left(\mathbf{r}, \mathbf{r}^{\prime}\right)$ are necessary. Thus, we mapped the original problem with random boundary condition onto the effective problem specified by the differential equation:

$$
\left(\nabla^{2}-s^{2}\right) \mathcal{G}_{s p}\left(r, r^{\prime}, \theta, \theta^{\prime}\right)=-\frac{2 m i \delta\left(r-r^{\prime}\right) \delta\left(\theta-\theta^{\prime}\right)}{r}
$$

and the uniform boundary condition

$$
\left.\frac{\partial}{\partial r} \mathcal{G}_{s p}\left(r, r^{\prime}, \theta, \theta^{\prime}\right)\right|_{S^{-}}=\left.i \frac{b \widetilde{Q}_{0}}{R} \mathcal{G}_{s p}\left(r, r^{\prime}, \theta, \theta^{\prime}\right)\right|_{S^{-}},
$$


where $s^{2}=-2 m E, b=m^{2} a v x$, and $S^{-}$is the inner surface of the dot. For both inner $(r<R)$ and outer $(r>R)$ domains the solutions are readily obtained and matched together. Below we will only need the solution for the inner domain, which can be written in terms of the modified Bessel functions $I_{n}$ and $K_{n}$ of $n$th order [19]:

$$
\begin{aligned}
\mathcal{G}_{s p}\left(\mathbf{r}, \mathbf{r}^{\prime}, s^{2}\right) & =\frac{i m}{\pi} \sum_{l} I_{l}\left(s r_{<}\right) \\
\times & \left\{a_{l} I_{l}\left(s r_{>}\right)+K_{l}\left(s r_{>}\right)\right\} \mathrm{e}^{i l\left(\theta-\theta^{\prime}\right)},
\end{aligned}
$$

and coefficients $a_{l}$ are given via

$$
a_{l}=\frac{-i b Q_{0}^{\prime} K_{l}(s R)+s R K_{l}^{\prime}(s R)}{i b Q_{0}^{\prime} I_{l}(s R)-s R I_{l}^{\prime}(s R)} .
$$

Then, $Q_{0}$ is obtained from the stationary point condition (Eq. (5)). Dropping the imaginary part of $\widetilde{Q}_{0}$, since it can be absorbed into $E$, we get

$$
\sum_{n} \frac{I_{n}^{2}(\widetilde{g})}{\left(f I_{n}(\widetilde{g})\right)^{2}+\left(\widetilde{g} I_{n}^{\prime}(\widetilde{g})\right)^{2}}=\frac{2 \pi\left(1+\frac{\bar{\gamma}}{2 x m d \widetilde{Q}_{0}}\right)}{x v b}
$$

Next, we set $\widetilde{g}=s R, f=b \widetilde{Q}_{0}$, and evaluate the sum over $n$ in Eq. (9) asymptotically in the following limit: $\widetilde{g} \gg 1$, $f / \widetilde{g} \sim 1$. We replace the sum with the integral, switch to the new variable $\mu=\nu / \widetilde{g}$, use uniform expansion for the Bessel function $I_{\nu}(\nu / \mu)$ 20 and expand the integrand in $1 / \widetilde{g}$; see Ref. 19] for the details. After the substitution $\widetilde{g} \rightarrow-i g(s \rightarrow-i k)$ the left-hand side of Eq. (9) becomes $\pi / 2 \sqrt{f^{2}-g^{2}}$ to the leading order in $1 / g$. Therefore, we have an algebraic equation for $f$ (or $\widetilde{Q}_{0}$ ), which can be solved with the help of the condition $k^{2}-(\pi / d)^{2}=m^{2} v^{2}$

At this point we introduce several relevant energy scales in "natural" units $\hbar=c=1$ : Thouless energy $E_{T}=k /(m R)$, mean level spacing of a closed $\operatorname{dot} \Delta=$ $1 /\left(m R^{2}\right)$, and a total resonance width $\Gamma=\bar{\gamma} /(m d R)$ related to the modal decay rate of a dot having identical coupling coefficients $\bar{\gamma}$ to all $N$ channels. In our almost closed dot we have the following hierarchy of scales: $E_{T} \gg \Delta \gg \Gamma$. Therefore, the set of dimensionless parameters specifying the problem completely is given by $g=E_{T} / \Delta=k R \gg 1$ (dimensionless conductance), $\mathcal{M}=\Gamma / \Delta \ll 1$ (modal overlap) together with $\bar{\gamma}, x$ and $v$. Any other constants which enters Eqs. (4 15), can be expressed in terms of these five. Hereafter we continue to use the old set of parameters, including $f$ and $\widetilde{Q}_{0}$, to keep the notation compact. The energy scales we just specified are to be used in comparison of our predictions to experimental and numerical results.

Now, we turn to the fluctuations around the saddle point, which can be decomposed into a transverse piece $\delta Q^{(t)}$ (along the saddle point manifold [9]) and a longitudinal piece $\delta Q^{(l)}$ (orthogonal to the saddle point manifold). The part of the action corresponding to the $\delta Q^{(t)}$ (anticommuting with $Q$ ) is given by 21 ]

$$
\begin{aligned}
F_{t}[\delta Q] & =-m^{2} a \int_{0}^{2 \pi}\left(\delta Q^{(t)}(\theta)\right)^{2} d \theta+\left(\frac{m x v a}{2}\right)^{2} \\
& \times \int_{0}^{2 \pi} \int_{0}^{2 \pi} \mathcal{G}(Q) \mathcal{G}(-Q) \delta Q^{(t)}(\theta) \delta Q^{(t)}\left(\theta^{\prime}\right) d \theta d \theta^{\prime} .
\end{aligned}
$$

Expanding in angular harmonics $\delta Q^{(t)}(\theta)=$ $\sum_{l=-\infty}^{\infty} Q_{l}^{(t)} \exp \{i l \theta\} / 2 \pi$ and using the Ward identity (relation between product and difference of $\mathcal{G}(Q)$ and $\mathcal{G}(-Q)$ ) it is possible to show that the massive term in $F_{t}[\delta Q]$ is proportional to $m R \bar{\gamma} / \pi x \widetilde{Q}_{0} \ll 1$. Then, applying the same technique, as in the solution of Eq. (9), we find that, to the leading order in $1 / g$, the free energy is quadratic in $\delta Q^{(t)}(\theta)$ for vanishingly small $\epsilon$, and arrive at

$$
\begin{gathered}
F_{t}[\delta Q] \simeq-D_{0} \int_{0}^{2 \pi}\left(\frac{\partial \delta Q^{(t)}}{\partial \theta}\right)^{2} d \theta \\
D_{0}=\left(\frac{m x v a \pi}{4 \widetilde{Q}_{0}}\right) \frac{g\left(2 g^{2}+f^{2}\right)}{f^{3} \sqrt{f^{2}-g^{2}}} .
\end{gathered}
$$

To finish the construction of the nonlinear $\sigma$-model, we integrate out the longitudinal modes, which decouple at this order in $1 / g$ from the transverse ones, and set $\delta Q^{(t)}=Q$. Next, we expand the symmetry breaking terms $F_{\epsilon}[\mathcal{G}(Q)]$ to the lowest order in $\epsilon$. The result is given by $\langle Z[J]\rangle_{\gamma}=\int D Q \mathrm{e}^{-F[Q]}$, with the free energy

$$
\begin{aligned}
F[Q] & =\operatorname{Str} \int d \mathbf{r} d \mathbf{r}^{\prime}\left\{D_{0}\left(\frac{\partial Q}{\partial \theta}\right)^{2} \frac{\delta(r-R)}{r} \delta\left(\mathbf{r}-\mathbf{r}^{\prime}\right)\right. \\
& \left.+\int d \theta^{\prime \prime} \epsilon \Lambda Q\left(\theta^{\prime \prime}\right) a\left(\mathbf{r}, R, \theta^{\prime \prime} ; \mathbf{r}^{\prime}, R, \theta^{\prime \prime}\right)\right\}
\end{aligned}
$$

$a\left(\mathbf{r}, R, \theta^{\prime \prime} ; \mathbf{r}^{\prime}, R, \theta^{\prime \prime}\right)=i \frac{m d x v}{2} \mathcal{G}_{s p}\left(R, \theta^{\prime \prime}, \mathbf{r}^{\prime}\right) \mathcal{G}_{s p}\left(\mathbf{r}, R, \theta^{\prime \prime}\right)$

where $r_{>}\left(r_{<}\right)$is a maximum (minimum) of $|\mathbf{r}|$ and $\left|\mathbf{r}^{\prime}\right|$. The supermatrix $Q$ satisfies a nonlinear constraint $Q^{2}=Q_{0}^{2} I_{4}$ and can be parametrized as suggested in Refs. 9, 17) for the diffusive case. The $n$-point correlations can be generated from the functional given by Eq. (10), which is the main result of this paper. Just as in case of the supersymmetric nonlinear $\sigma$-model of Ref. 9], the diffusion modes clearly play an important role in the superintegrals representing correlators.

A physically measurable quantity which does not depend on fluctuations around the saddle point is average local density of states (LDOS) $\langle\rho(\mathbf{r})\rangle_{\gamma}=$ $-(1 / \pi) \Im\left\langle G\left(\mathbf{r}, \mathbf{r}^{\prime}, E\right)\right\rangle_{\gamma}$. Our Indeed, this one-point function neither requires the knowledge of the $\Lambda$ like structure of the saddle point manifold, nor its explicit parametrization. It can be shown that, 
$\Im\left\langle G\left(\mathbf{r}, \mathbf{r}^{\prime}, E\right)\right\rangle_{\gamma}=\left.\Im\left\langle\mathcal{G}\left(r, r^{\prime}, \theta, \theta^{\prime}, s^{2}+i \epsilon\right)\right\rangle_{Q}\right|_{s=-i k}$, where $\langle\ldots\rangle_{Q}$ stands for integration with weight $\exp \{-F[Q]\}$ (Eq. (10) ). This integration reduces to evaluating the integrand at the saddle point. Most conveniently, the average LDOS can be calculated via regularized resolvent $\widetilde{K}\left(r, s^{2}\right)=\mathcal{G}\left(r, r, \theta, \theta, s^{2}\right)-$ $\mathcal{G}_{0}\left(r, r, \theta, \theta, s^{2}\right)[19]: \pi\langle\rho(\mathbf{r})\rangle_{\gamma}=m+\left.\Im \widetilde{K}\left(r, s^{2}\right)\right|_{s=-i k}$. The result reads

$$
\Im \widetilde{K}\left(s^{2}\right)=\frac{m}{\pi} f \sum_{n} \frac{I_{n}^{2}(\kappa g)}{f^{2} I_{n}^{2}(g)+g^{2}\left(I_{n}^{\prime}(g)\right)^{2}},
$$

where $\kappa=r / R$. This expression for $\Im \widetilde{K}\left(s^{2}\right)$ can be further simplified for the limiting cases: (i) $\kappa \ll 1 / g$, when the main contribution to sum in Eq. (11) comes from the terms with small $n$, and (ii) $\kappa \gtrsim 1 / g$, when we can employ the uniform expansions of $I_{n}$. The results are summarized in Fig. 1]

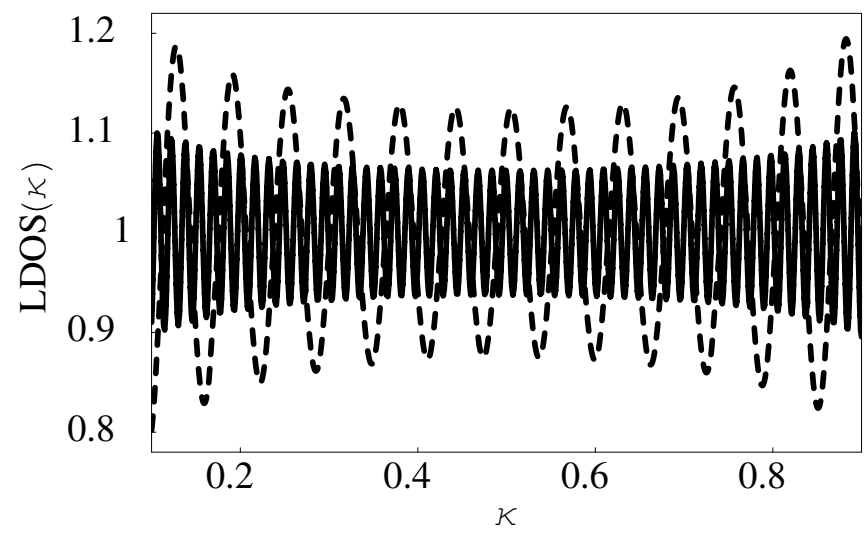

Figure 1: LDOS plotted as a function of $\kappa$ in units of $m / \pi$ for two sets of parameters: $g=50, \mathcal{M}=1 / 12$ (dashed line); and $g=200, \mathcal{M}=1 / 3$ (solid line). In both cases the rest of the parameters were fixed $x=0.1 ; \bar{\gamma}=0.2 ; v=100$;

Thus, we have constructed a nonperturbative theoretical framework to analyze one particular realization of a whole class of nanostructures: a nearly closed system with ballistic internal dynamics interacting randomly with the outside world through the boundary. Our approach introduces a natural regularizer, which enables us to circumvent the conceptual difficulties of previous approaches to closed ballistic systems [10]. We find that the resulting theory, encapsulated by Eq. (10), can be characterized by diffusive modes confined to the boundary and interacting nonlocally with the interior (see the last term of Eq. (10)). The supersymmetric functional was constructed with the help of large angular momentum modes identified as the WGM. These modes are exponentially less likely to escape [13] compared to the modes with incidence directions close to the lead normals, and consequently, they dominate response functions at large times. Our framework should allow us to compute the statistical properties of any physically measurable quantity, though technical difficulties may impose strong limits. It should be clear that our approach is also applicable, with minor modifications, to other examples belonging to this class of systems.

Finally, the extension of this approach to generic billiards with smooth walls (to be published elsewhere) is also possible, although it is more technically involved 26]. At first sight the non-linear supersymmetric $\sigma$ model (NLS $\sigma \mathrm{M})$ for the rough billiards proposed in Ref. 22] looks very similar to the NLS $\sigma$ Ms we derived here for open circular billiard (and for open rough billiard in Ref. 26]). However, there are several differences between two models, which can be summarized as follows. The diffusion and (one-dimensional) localization in angular momentum $l$ space described in Ref. [22] is guaranteed by small changes in $l$ as the particle bounces off the walls. In our case, because of the sharp edges of the region which connects the leads to the dot the WGM trajectories may have much larger $l$ increments along the way. As a result our model describes diffusion and localization in position (angle $\theta$ ) space rather than angular momentum space.

Another issue is the role of electronic interactions. One of the possible ways to take them into account in diffusive and ballistic systems with large dimensionless conductance, is to use a "Universal Hamiltonian" [23], which was shown to be the renormalization group fixed point for weak interactions $[24,25]$. We hope to extend our analysis to the interacting ballistic case by using the large- $N$ approach of Ref. 25]. We leave these questions for future work.

We are grateful to the NSF for partial support under DMR-0311761.

[1] Kouwenhoven L P et al., in Proceedings of NATO ASI on Mesoscopic Electronic Transport edited by L.L. Sohn, G. Sohn, and Kouwenhoven L P, 105 (Kluwer, Dordrecht 1997).

[2] D. K. Ferry and S. M. Goodnick. Transport in nanostructures (Cambridge University Press, 1997).

[3] A. Altland, C.R. Offer, and B.D. Simons, in Supersymmetry and Trace Formulae, edited by I.V. Lerner, J.P. Keating, and D.E. Khmelnitskii (Kluwer, Dordrecht, 1999).

[4] G.A. Fiete and E.J. Heller, Rev. Mod. Phys. 75, 933 (2003).

[5] Colas des Francs et al., Phys. Rev. Lett. 86, 4950 (2001).

[6] Turechi H E et al. e-print: physics/0308016

[7] Y. Decanini and A. Folacci, Phys. Rev. A 67, 042704 (2003).

[8] C. H. Lewenkopf and A. Müller and E. Doron, Phys. Rev. A 45, 2635 (1992); for the recent review see Y. V. Fyodorov, D. V. Savin and H.-J. Sommers, e-print: cond-mat/0507016

[9] K. Efetov. Supersymmetry in disorder and chaos (Cambridge University Press, 1997). 
[10] Y.M. Blanter, A.D. Mirlin, and B.A. Muzikantskii, Phys. Rev. B 63, 235315 (2001); K.B. Efetov and V.R. Kogan, Phys. Rev. B 67, 245312 (2003); K.B. Efetov, G. Schwiete, and K. Takahashi, Phys. Rev. Lett. 92, 026807 (2004).

[11] R.G Nazmitdinov et al., Phys. Rev. E 64, 056214 (2001); R.G Nazmitdinov et al., Phys. Rev. B 66, 085322 (2002).

[12] I. L. Aleiner and A. I. Larkin, Phys. Rev. E 55, R1243 (1997); K. Richter and M. Sieber, Phys. Rev. Lett 89, 206801 (2002).

[13] C.D. Schwieters, J.A. Alford and J.B. Delos, Phys. Rev. B, 54, 10652 (1996).

[14] Chapter 9 of Ref. [9].

[15] S. Iida, H. A. Weidenmüller and J. A. Zuk, Ann. Phys. (NY) 200, 219 (1990); for a nice review see Y.V. Fyodorov, H.-J. Sommers, J. Math. Phys. 38, 1918 (1997); F.-M. Dittes, Phys. Rep. 339, 215 (2000).

[16] C.H. Lewenkopf, A. Müller, and E. Doron, Phys. Rev. A 45, 2635 (1992); K. Pichugin, H. Schanz, and P. Seba, Phys. Rev. E 64, 056227 (2001); R.A. Mendez-Sanchez et al., Phys. Rev. Lett. 91, 174102 (2003); O.I. Lobkis, I.S. Rozhkov, and R.L. Weaver, ibid. 91, 194101 (2003).

[17] A. Mirlin, Phys. Rep. 326, 259 (2000); Proceedings of the International School of Physics "Enrico Fermi" on New Directions in Quantum Chaos, eds. G. Casati, I. Guarneri, and U. Smilansky (IOS Press Amsterdam, 2000), 223.

[18] To generate averages of products of $G^{R, A}\left(\mathbf{r}, \mathbf{r}^{\prime}\right)$, which is usually the goal in any statistical analysis, one needs to introduce source terms into action $\mathcal{L}$, such as $\int \Psi^{\dagger}(\mathbf{r}) J(\mathbf{r}) \Psi(\mathbf{r}) d \mathbf{r}$, with $J(\mathbf{r})$ being a coordinate dependent supermatrix, or $\int \Psi^{\dagger}(\mathbf{r}) J(\mathbf{r})+J^{\dagger}(\mathbf{r}) \Psi(\mathbf{r}) d \mathbf{r}$, with $J(\mathbf{r})$ being a coordinate dependent supervector. These terms are not important in the derivation of the $\sigma$-model, and we ignore them in the process.

[19] K. Stewartson and R.T. Waechter, Proc. Camb. Phil. Soc. 69, 353 (1971); M. Sieber et al. J. Phys. A 28, 5041 (1995).

[20] M. Abramowitz and I.A. Stegun. Handbook of mathematical functions (New York, Dover, 1965).

[21] Here we omit the variation of $F_{\epsilon}[\mathcal{G}]$. It is easily restored in the end.

[22] K. M. Frahm, Phys. Rev. B 55, R8626 (1997).

[23] A.V. Andreev and A. Kamenev, Phys. Rev. Lett. 81, 3199 (1998); P.W. Brouwer, Y. Oreg, and B.I. Halperin, Phys. Rev. B, 60, R13977 (1999); H.U. Baranger, D. Ullmo, and L.I. Glazman, ibid. 61, R2425 (2000); I.L. Kurland, I.L. Aleiner, and B.L. Altshuler, ibid. 62, 14886 (2000).

[24] G. Murthy and H. Mathur, Phys. Rev. Lett. 89, 126804 (2002).

[25] G. Murthy et al., Phys. Rev. B 69, 075321 (2004).

[26] Igor Rozhkov and G. Murthy, e-print: cond-mat/0507186 